\title{
DAS SELBSTREFLEXIVE ICH-SUBJEKT ALS ANGELPUNKT RATIONALER THEOLOGIE BEI HOLM TETENS
}

Fabian Müller

DOI 10.32701/dp.20.1.4

Der emeritierte Berliner Philosoph Holm Tetens hat sich während seiner akademischen Tätigkeit intensiv mit dem Naturalismus auseinandergesetzt. In den letzten Jahren aber hat er sich vor allem religionsphilosophischen und theologischen Themen zugewandt. In seinem 2015 erschienenen Buch ist Tetens erstmals für einen »theistischen Idealismus « ${ }^{1}$ eingetreten, der besagt, dass »Gott als unendliches Ich-Subjekt «² existiert, der den Menschen schafft und sein Heil will. ${ }^{3}$ Tetens legt keinen für sich stehenden Beweis für den Theismus vor, sondern argumentiert dafür, dass der Theismus vernünftiger als der Naturalismus sei. Bei diesem komparativen Argumentationsgang dient das selbstreflexive Ich-Subjekt als wichtiges Entscheidungskriterium, um zwischen den beiden metaphysischen Positionen auszuwählen. In diesem Artikel soll zunächst Tetens‘ Argumentation für eine rationale Theologie nachgezeichnet und dann auf dem Hintergrund wissenschaftsphilosophischer Überlegungen auf das selbstreflexive Ich-Subjekt eingegangen werden.

Tetens konstatiert zunächst, dass jeder Mensch mit bestimmten Fragen unmittelbar konfrontiert ist: »Wie konnten in einem vermeintlich anfänglich rein materiellen physikalischen Universum schließlich wir Menschen als selbstreflexive Ich-Subjekte in Erscheinung treten? « ${ }^{4}$ Warum müssen wir sterben? Was bedeutet der Tod? $«^{5} »$ Was ist mit den unglaublich vielen unver-

* $\quad$ Fabian Müller, Dissertant am Institut für Dogmatik / Doctoral candidate at the Institute for Dogmatics, University of Graz, E-mail: fabian.mueller@edu.uni-graz.at

1 Holm Tetens, Gott denken. Ein Versuch über rationale Theologie (Stuttgart: Reclam, 2015), 80.

2 Ebd.

3 Ebd.

4 Holm Tetens, »Gott als Antwort auf Fragen, die wir nicht loswerden, « In Gott denken. Zur Philosophie von Religion, hg. Christoph Böhr und Hanna-Babara Gerl-Falkovitz (Wiesbaden: Springer, 2018), 276. Den Begriff »selbstreflexives Ich-Subjekt« werden wir noch entfalten. Ebd., 277. 
söhnt und ungetröstet verstorbenen Opfern der Weltgeschichte? Ist die Chance unwiderruflich vorbei, sie zu trösten und ihnen doch noch Gerechtigkeit widerfahren $\mathrm{zu}$ lassen? ${ }^{6}$ Auf solche grundlegenden Fragen eine Antwort zu geben, kommt vor allem der Metaphysik ${ }^{7}$ zu. Die heute vorherrschende Metaphysik ist der Naturalismus. Dieser behauptet: »Die eigentliche und einzige Wirklichkeit ist die Welt materieller Dinge und Ereignisse, wie sie im Wesentlichen zutreffend durch die Naturwissenschaften beschrieben und erklärt werden «. ${ }^{8}$ Die vorhin angeführten Fragen sind dann nicht beantwortbar. ${ }^{9}$ Postuliert man eine Beantwortbarkeit dieser Fragen, dann muss man das Dasein und Wirken Gottes mitdenken. Nur wenn man annimmt, dass Gott existiert und wirkt, kann man darauf hoffen, dass beispielsweise den unzähligen Opfern der Weltgeschichte doch noch Gerechtigkeit widerfahren wird. Das Postulat positiver Antworten auf die angeführten Fragen und das Postulat des Theismus sind aussagenlogisch äquivalent. ${ }^{10}$ Der Theismus kann damit Fragen beantworten, die der Naturalismus nicht beantworten kann. Da der Naturalismus überdies nicht bewiesen ist, ist es für Tetens vernünftiger »im Leben auf Gott als Schöpfer und Erlöser der Welt zu hoffen, als naturalistisch davon auszugehen, [...] dass wir im Leben und im Sterben ganz auf uns allein gestellt seien « ${ }^{11}$. Die Argumentation von Tetens lässt sich in vereinfachter Form wie folgt darstellen:

Prämisse 1: Es gibt bestimmte existentielle Fragen, die sich dem Menschen unausweichlich stellen.

Prämisse 2: Der Theismus kann die existentiellen Fragen beantworten.

Prämisse 3: Der Naturalismus kann die existentiellen Fragen nicht beantworten. Prämisse 4: Der Theismus ist nicht bewiesen.

Prämisse 5: Der Naturalismus ist nicht bewiesen.

Conclusio: Der Theismus ist vorzuziehen.

In der folgenden Stellungnahme zu Tetens setze ich voraus, dass es tatsächlich bestimmte existentielle Fragen gibt, denen der Mensch nicht entkommt. Es wird weiters zugestanden, dass der Theismus in der Lage ist, eine positive Antwort auf die existentiellen Fragen zu geben, der Naturalismus hingegen nicht. Weiters möchte ich die Einschränkung auf den Theismus und Naturalismus beibehalten und die Frage, ob nicht auch andere Positionen, also andere Metaphysiken eine Antwort auf existentielle Fragen ge-

6 Ebd.

7 Metaphysik meint bei Tetens vor allem eine bestimmte Ontologie. Sie beantwortet die Frage, welche Dinge es gibt und welche Dinge lediglich von den Dingen, die es gibt, abgeleitet sind.

8 Tetens, Gott denken, 80.

9 Tetens, Gott als Antwort, 281.

10 Ebd. 280 (Nr.3.3. und 3.4).

11 Ebd., 293. 
ben könnten, ausklammern. Es sollen lediglich zwei Fragen geklärt werden: erstens, inwiefern die Prämisse von der Unbeweisbarkeit des Naturalismus zutrifft (Prämisse 5) und zweitens, ob die Conclusio, der Theismus sei vorzuziehen, folgerichtig ist.

Der Naturalismus, so Tetens, ist eine metaphysische Position, die aus den Resultaten der Naturwissenschaften nicht zwingend folgt, auch wenn dies oft behauptet werde. ${ }^{12}$ Da jede Metaphysik mit Allsätzen arbeitet und Allsätze sich nie, auch durch noch so viele empirische Belege, beweisen lassen, kann keine Metaphysik durch empirische Belege bewiesen werden (Induktionsproblem). ${ }^{13}$

»Ist der Naturalismus wahr? Jedenfalls ist er nicht bewiesen. Zwar tun viele Naturalisten unter den Philosophen so, als ob der Naturalismus logisch zwingend aus den Ergebnissen der empirischen Wissenschaften folgen würde. Das ist jedoch falsch. Die Ergebnisse der Naturwissenschaften implizieren nur den Satz: Es gibt die Erfahrungswelt, wie sie mit den methodischen Mitteln der empirischen Wissenschaften erfolgreich beschrieben und erklärt werden kann « ${ }^{14}$.

Einerseits möchte Tetens die überhöhten Ansprüche von Naturalisten, wie die Behauptung, es gäbe nur die naturwissenschaftlich erfassbare Erfahrungswelt, zurückweisen..$^{15}$ Andererseits gesteht er aber, wie aus dem oben angeführten Zitat hervorgeht, zu, dass aus den Ergebnissen der Naturwissenschaften folgt: Es gibt die naturwissenschaftliche erfassbare Erfahrungswelt. Für mich ist unverständlich geblieben, wie aus den Ergebnissen der Naturwissenschaften auf Existenzaussagen geschlossen werden soll. Tetens statuiert, dass »kein vernünftiger Mensch « ${ }^{16}$ die Existenz der Erfahrungswelt bezweifelt. Nur folgt dies, soweit ich sehe, nicht aus den Ergebnissen der Naturwissenschaften, sondern der Forscher muss die ontologische Verpflichtung eingehen, damit die Ergebnisse der naturwissenschaftlichen Disziplinen wahr sein können. So kann eine Theorie über Elektronen dem Beobachter nur dann etwas Wahres sagen, wenn er bereit ist, zu akzeptieren, dass es Elektronen wirklich gibt. Die Theorie kann die Existenz von Elektronen nicht beweisen, sondern muss sie bereits voraussetzen. In jeder Theorie und in jeder Metaphysik muss man gewisse Entitäten postulieren, die es tatsächlich geben muss, damit die Theorie bzw. Metaphysik als wahr angesehen werden kann. ${ }^{17}$ Davon unberührt ist die Feststellung, dass »kein

Tetens, Gott denken, 90.

Ebd., 18.

Tetens, Gott als Antwort, 279.

Ebd.

Ebd.

Phillip Bricker, »Ontological Commitment. Version vom 03.11.2014,« in Stanford Encyclopedia of Philosophy, hg. Edward N. Zalta. https://plato.stanford.edu/entries/ontological-commitment/, 1.5 (PDF-Version). 
vernünftiger Mensch« zögern wird, die ontologische Verpflichtung in Bezug auf die Erfahrungswelt einzugehen.

An anderer Stelle räumt Tetens ein, dass man am Theismus festhalten sollte, »es sei denn, das Überzeugungssystem Z [= Naturalismus] ist bewiesen oder es sprechen andere gewichtige Gründe gegen das Überzeugungssystem Y [= Theismus] « ${ }^{18}$. In guter wissenschaftstheoretischer Manier lässt Tetens hier die Möglichkeit offen, dass in Zukunft der Naturalismus doch noch bewiesen werden könnte. Gewiss ist die Zukunft offen und weitgehend unvorhersehbar, doch sehe ich hier eine gewisse Spannung zu Tetens Beschreibung der Grundstruktur von Metaphysik. »Die Kernbehauptung einer jeden Metaphysik ist ein Allsatz. Er lässt sich nicht durch Erfahrung beweisen $^{19}$. Es ist mir dabei unerklärlich geblieben, wie ein Überzeugungssystem, also eine Metaphysik, in Zukunft einmal bewiesen werden könnte. Es ist m.E. im Gegenteil davon auszugehen, dass keine Metaphysik, da sie mit unbeweisbaren Allsätzen arbeitet, jemals bewiesen werden kann (Induktionsproblem). Weiters machen unsere vorherigen Überlegungen deutlich, dass jede Metaphysik, da sie mit Existenzaussagen arbeitet, gewisse ontologische Verpflichtungen voraussetzt, die der Forscher eingehen kann oder nicht. Tetens selbst legt ja am Beginn seiner Ausführungen dar, dass er unterstellen muss, dass »die Erfahrungswelt im Wesentlichen genauso ist, wie die Wissenschaften sie beschreiben und erklären $\ll^{20}$. Ist eine Metaphysik nun grundsätzlich nicht beweisbar, so wird sie dies auch in Zukunft nicht sein. Die hier vorgebrachte Kritik an Tetens im Einzelnen ändert jedoch nichts an seiner Grundthese »Der Naturalismus ist nicht beweisbar«, sondern bestätigt sie viel mehr.

Tetens geht implizit davon aus, dass der Theismus unbeweisbar ist, ebenso hält er den Naturalismus für unbeweisbar. Es scheint sich eine Pattsituation anzubahnen. Doch für Tetens gibt es ein Kriterium, das bei der Entscheidungsfindung einen signifikanten Beitrag zu leisten vermag.

»Nur wenn sich die Sinnfragen anthropologisch positiv beantworten lassen, dürfen wir uns selbst uneingeschränkt und ohne kognitive und moralische Brüche und Dissonanzen als zwar endliche, aber doch vernünftige, selbstbestimmte, selbstverantwortliche, auf das Wahre, Gute und Schöne hin orientierte Ich-Subjekte in einer materiellen Erfahrungswelt verstehen und begreifen. Und eigentlich wollen wir uns so verstehen ${ }^{21}$.

Mit dieser Beschreibung des Menschen legt Tetens eine ganz bestimmte philosophische Anthropologie vor. Er statuiert, dass jeder Mensch auf etwas 
hin orientiert ist und sich als selbstreflexives Ich-Subjekt verstehen möchte. Man könnte dieses Streben als eine anthropologische Grundkonstante bezeichnen. Daher »sollten wir überwältigend gute Evidenzen anführen können, bevor wir das besagte Selbstverständnis als vermeintlich illusionär fallen lassen $\varkappa^{22}$. Somit bettet Tetens den vorhin statuierten Wunsch des Menschen in eine normative Aussage ein. Der Mensch möchte sich nicht nur als selbstreflexives Ich-Subjekt verstehen, sondern er soll es auch. Dies ist ein entscheidender Schritt innerhalb des Projektes der rationalen Theologie. Aus den vorhin angeführten fünf Prämissen und der eben genannten Norm lässt sich dann folgern: Es ist »vernünftiger [...] im Leben auf Gott [...] zu hoffen, als naturalistisch davon auszugehen, [...] dass wir im Leben und im Sterben ganz auf uns allein gestellt sind « ${ }^{23}$. Auf der Grundlage unserer Überlegungen lässt sich das Argument von Tetens nun in präzisierter Form notieren:

Prämisse 1: Es gibt bestimmte existentielle Fragen, die sich dem Menschen unausweichlich stellen.

Prämisse 2: Das Selbstverständnis des Menschen als selbstreflexives Ich-Subjekt ermöglicht eine positive Beantwortung existentieller Fragen.

Prämisse 3: Der Theismus erlaubt es dem Menschen, sich als selbstreflexives Ich-Subjekt zu verstehen.

Prämisse 4: Der Naturalismus erlaubt dies nicht.

Prämisse 5: Der Theismus ist nicht bewiesen.

Prämisse 6: Der Naturalismus ist nicht bewiesen.

Norm: Der Mensch soll sich als selbstreflexives Ich-Subjekt verstehen.

Conclusio: Der Theismus ist vernünftiger.

In der vorliegenden Form ist Tetens‘ Argument folgerichtig. Das heißt die Conclusio folgt aus den Prämissen, einzig das Wort »vernünftiger« schafft noch Probleme. Der Vernunftbegriff bei Tetens hat eine explizit normative Dimension. ${ }^{24}$ Eine Metaphysik ist dann vernünftig, wenn sie dem Menschen erlaubt, sich als selbstreflexives Ich-Subjekt zu verstehen. Hier steckt im Vernunftbegriff eine ganz bestimmte philosophische Anthropologie; erst wenn man ein solches Verständnis von Vernunft hat, ist das oben angeführte Argument folgerichtig.

Das Einfügen einer normativen Prämisse in ein ansonsten deskriptives Argument birgt natürlich auch Risiken, da es gewiss eine ganze Reihe von Metaphysiken gibt, die es dem Menschen möglich machen, sich als selbstreflexives Ich-Subjekt zu verstehen. Demnach könnte er einfach nach Gutdünken eine bestimmte Metaphysik auswählen. Diese offene Flanke seines

22 Ebd., 292.

23 Tetens, Gott als Antwort, 293.

24 Wie Tetens selbst ausführt, folgt er hier der Position Kants (Kritik der praktischen Vernunft). Tetens, Gott als Antwort, 294 (Fußnote 19). 
Argumentes versucht Tetens durch das Einführen dreier Normen zu schließen. Er spricht hier zwar von Normen für rationale Theologie, man darf aber annehmen, dass diese Normen für jede Metaphysik gelten sollen. Erstens darf keine Metaphysik den »anerkannten Thesen der [Natur-]Wissenschaften widersprechen $\ll^{25}$. Zweitens darf sie keinen Wunderglauben beinhalten und drittens muss sich jede Metaphysik an die Gesetze der Logik halten ${ }^{26}$. Damit wird ein willkürliches Auswählen einer dem Menschen genehmen Metaphysik verhindert. Eine ganze Reihe an metaphysischen Modellen kann diesem Kriterium nicht genügen. So ist z.B. ein Theismus, der das Alter der Erde auf etwa 6000 Jahre festsetzt oder Adam und Eva im genealogischen Sinne als die ersten Menschen der Geschichte versteht, vom Tisch, da er anerkannten Ergebnissen der empirischen Forschung widerspricht. Ebenso ist Gott an die Gesetze der Logik gebunden, er kann keine verheirateten Junggesellen erschaffen. ${ }^{27}$ Von den drei angeführten Normen, ist für unsere Fragestellung vor allem die erste von Relevanz: Eine Metaphysik darf den anerkannten Ergebnissen der empirischen Forschung nicht widersprechen.

Metaphysiken anhand bestimmter Normen und nicht nach erkenntnistheoretischen Parametern auszuwählen, mag auf den ersten Blick als ein gewagter Schritt erscheinen, doch ist dies in vielen wissenschaftsphilosophischen Disziplinen eine anerkannte Vorgehensweise. So kennt schon Wilhelm von Ockham (1285-1347) die Norm, Theorien so einfach wie möglich zu formulieren. Gibt es zwei Theorien, die beide durch empirische Daten bestätigt werden, so gilt es jene zu verwenden, die einfacher ist bzw. mit weniger postulierten Entitäten auskommt ${ }^{28}$. Die Norm der Einfachheit gilt gewöhnlich bei der Auswahl zweier Theorien. Tetens verhandelt hier hingegen ganze Metaphysiken. Dennoch besteht eine gewisse Ähnlichkeit zwischen Ockhams Norm der Einfachheit und Tetens' Normen ${ }^{29}$. Beide Male sind bei der Auswahl von zwei Theorien bzw. Metaphysiken nicht nur empirische Daten ausschlaggebend, sondern auch Normen. Die Norm der Einfachheit erleichtert den Umgang mit der Welt, in der der Mensch lebt. Sie

Tetens, Gott denken, 8.

Tetens, Gott denken, 8

Ebd. Ob Gottes Allmacht durch die Gesetze der Logik beschränkt ist, war vor allem im Mittelalter eine intensiv diskutierte Frage. Laut Thomas von Aquin (1225-1274) ist Gott an die Logik gebunden. Brian Leftow, »God's omnipotence, « in Oxford Handbook of Aquinas, ed. Brian Davies and Eleonore Stump (Oxford: Oxford University Press, 2012), 187-195.

Vgl. Alan Baker, »Simplicity. Version vom 20.12.2016, « in Stanford Encyclopedia of Philosophy, hg. Edward N. Zalta. https://plato.stanford.edu/entries/simplicity/, 2-6 (PDF-Version).

Dabei sind die Unterschiede ebenfalls deutlich: Die Auswahl findet hier zwischen unterschiedlichen Theorien statt, dort zwischen unterschiedlichen Metaphysiken. Hier sind zwei Theorien durch empirische Daten im gleichen Maße bestätigt, dort stehen sie lediglich in keinem Widerspruch zur Theorie (Metaphysik). Theorien lassen sich grundsätzlich durch empirische Daten bestätigen, bei Metaphysiken ist dies nicht möglich. 
lässt sich nicht auf erkenntnistheoretischem Wege rechtfertigen, sondern ist allein der Pragmatik geschuldet. Sie erleichtert den Alltag und das Leben des Menschen. Genau dies macht auch Tetens, wenn er mit Hilfe einer Norm zwischen zwei Metaphysiken auswählt. Da die Ergebnisse der empirischen Forschung keine der beiden Metaphysiken als ganze bestätigen können, soll der Forscher jene Metaphysik auswählen, die das Leben des Menschen erleichtert. Tetens spricht hier aber nicht nur von »erleichternd« oder »nützlicher«, sondern von »besser « ${ }^{30}$. Was für den Menschen gut oder schlecht ist, ergibt sich auf der Grundlage einer bestimmten philosophischen Anthropologie. An dieser Stelle überschreitet Tetens den Bereich, der sich durch den Vergleich mit Okcham's razor rechtfertigen ließe.

Obwohl Tetens die Norm, der Mensch solle sich als selbstreflexives IchSubjekt verstehen, durch die zweite Norm, dass keine Metaphysik den anerkannten Ergebnissen der Naturwissenschaften widersprechen darf, abfängt, bleibt dennoch das Problem bestehen, dass immer noch eine ganze Reihe von Metaphysiken sich so konstruieren lassen, dass sie beiden Normen gerecht werden können. Es haftet jeder Metaphysik also eine gewisse Beliebigkeit an. Dies gesteht auch Tetens zu, wenn er im Bezug auf die Metaphysik feststellt, dass »[i]mmer [...] ein Spielraum $\ll^{31}$ bleibt. Weiters könne jeder Forscher »den erkenntnistheoretischen Joker ziehen ${ }^{32}$ und behaupten, es existierten die Entitäten $\mathrm{x}$ und y, aber sie seien für uns nicht erkennbar. Dieses Problem ist auch in der Wissenschaftsphilosophie der Naturwissenschaften bekannt. So kann eine bestimmte Theorie gegen widersprechende empirische Daten immunisiert werden, indem man die Umgebungstheorien, in die diese Theorie eingebettet ist, verändert. So konnte z.B Issak Newton (1642-1724) an seiner Theorie über die Umlaufbahnen der Planeten festhalten, obwohl die Umlaufbahn des Merkur nicht seiner astronomischen Theorie entsprach. Dabei postulierte Newton eine ungleichmäßige Verteilung der Masse in der Sonne. Dieses grundlegende Problem ist unter dem Namen Duhem-Quine-These bekannt. ${ }^{33}$ Damit ist das Vorgehen von Tetens nicht begründet, da es aber ähnliche Überlegungen in vielen wissenschaftsphilosophischen Disziplinen gibt, ist aber gezeigt, dass er an dieser Stelle keinen Sonderweg beschreitet.

Der Komparativ »besser« kommt zwar nicht explizit vor, legt sich aber zusammenfassend nahe. Tetens, Gott als Antwort, 292-293.

31 Tetens, Gott denken, 18.

32 Ebd., 28.

33 Roberta Lanfredi, »Wissenschaftsphilosophie, « in Enzyklopädie Philosophie hg. v. Hans Jörg Sandkühler (Hamburg: Meiner, 2010), 3044.

Kyle Stanford, »Underdetermination of Scientific Theory. Version vom 12.10.2017, « in Stanford Encyclopedia of Philosophy, hg. Edward N. Zalta. https://plato.stanford.edu/entries/scientific-underdetermination/, 3-5 (PDF-Version). 
Die Stärke von Tetens' Ansatz besteht zweifellos darin, dass er keine zu ambitionierte These aufstellt. Er legt keinen neuen Gottesbeweis vor, der nur mit den Mitteln der Vernunft den christlichen Gott beweisen wollte. Tetens geht vom gegenwärtig vorherrschenden Weltbild, dem Naturalismus, aus, um dann zu zeigen, dass der Theismus im Vergleich vernünftiger ist. Dabei muss er mit Nachdruck für eine bestimmte philosophische Anthropologie werben und konstatieren, dass der Mensch sich als selbstreflexives Ich-Subjekt verstehen will und auch soll. Erst mit dieser Norm lässt sich auf vernünftige Weise zwischen zwei Metaphysiken auswählen. Einige seiner Argumentationsschritte sind zum Teil auch in wissenschaftsphilosophischen Disziplinen bekannt. An bestimmten Stellen (z.B. Ockham's razor) übersteigt Tetens aber den in der Wissenschaftsphilosophie bekannten Rahmen.

\section{Literaturverzeichnis}

Baker, Alan. »Simplicity. Version vom 20.12.2016. «in Stanford Encyclopedia of Philosophy, hg. Edward N. Zalta. https://plato.stanford.edu/entries/simplicity/.

Bricker, Phillip. »Ontological Commitment. Version vom 03.11.2014.« in Stanford Encyclopedia of Philosophy, herausgeben von Edward N. Zalta. https://plato. stanford.edu/entries/ontological-commitment/.

Lanfredi, Roberta. »Wissenschaftsphilosophie.« in Enzyklopädie Philosophie, herausgegeben von Hans Jörg Sandkühler, 3038-3048. Hamburg: Meiner, 2010.

Leftow, Brian. »God's omnipotence.« in Oxford Handbook of Aquinas, edited by Brian Davies and Eleonore Stump, 187-195. Oxford: Oxford University Press, 2012.

Stanford, Kyle. »Underdetermination of Scientific Theory. Version vom 12.10.2017.« in Stanford Encyclopedia of Philosophy, herausgeben von Edward N. Zalta. https://plato.stanford.edu/entries/scientific-underdetermination/.

Tetens, Holm. Gott denken. Ein Versuch über rationale Theologie. Stuttgart: Reclam, 2015.

Tetens, Holm. »Gott als Antwort auf Fragen, die wir nicht loswerden.« In Gott denken. Zur Philosophie von Religion, herausgegeben von Christoph Böhr und HannaBabara Gerl-Falkovitz, 275-294. Wiesbaden: Springer, 2018. 
Abstract

\section{DAS SELBSTREFLEXIVE ICH-SUBJEKT ALS ANGELPUNKT RATIONALER THEOLOGIE BEI HOLM TETENS}

Holm Tetens, a philosopher of the Freie Universität Berlin, reasons for the existence of God. In contrast to classical arguments for God's existence, he uses a comparative way of reasoning. Tetens starts with naturalism, the prevalent metaphysics of present times, and tries to show that in comparison to that, theism is the more reasonable choice. Neither naturalism nor theism can be proven. Thus, Tetens has to find different criterion in other to show that theism is more reasonable than naturalism. In his understanding, a human being is a morally responsible I-subject (selbstreflexives Ich-Subjekt). Unlike naturalism, theism is compatible with this philosophical anthropology, making it the more reasonable metaphysics. This article aims to trace back Tetens argument and examines his claim that no metaphysics can pe proven by empirical data. A special emphasis is laid on Tetens' concept of the morally responsible I-subject as the central criterion in order to choose between two metaphysics.

KEY wORDS: Holm Tetens; naturalism; theism; morally responsible I-subject; philosophy of science 